\title{
Factores que explican la intención de compra de pizzas en jóvenes universitarios de carreras de negocio en una universidad de Lima
}

\author{
Enrique Saravia Vergara \\ Candidato a Doctor en Sociedad de la Información y el Conocimiento \\ por la Universitat Oberta de Catalunya, España. \\ Master en Sociedad de la Información y el Conocimiento \\ por la Universitat Oberta de Catalunya, España. \\ Magíster en Administración por la Universidad del Pacífico, Lima, Perú. \\ Profesor e Investigador del Departamento Académico de Administración de la \\ Universidad del Pacífico, Lima, Perú.
}

\begin{abstract}
Resumen
El presente estudio explora las principales variables que influyen en la decisión de compra de los consumidores de pizza, en el segmento de jóvenes universitarios de carreras de negocio en una universidad de Lima. EI estudio muestra que la intención de compra de pizzas en este grupo objetivo depende de la «imagen» de la empresa, el «precio»y, en tercera instancia, la «calidad» de las pizzas, mientras que la «responsabilidad social» influye en estos atributos. A su vez, en la investigación también se identifican las variables relevantes de cada uno de estos factores, como el prestigio, la confianza y la garantía de la marca; el precio y las promociones y descuentos; el sabor y la calidad de los insumos; la respuesta a reclamos y sugerencias; la atractividad de los locales, entre otras variables relevantes.
\end{abstract}

Palabras clave: intención de compra de pizzas, factores de decisión de compra, atributos de calidad de pizzas, imagen empresarial, responsabilidad social.

\section{Introducción}

El mercado de pizzas en Lima es muy dinámico $y$, en la actualidad, existe una fuerte competencia entre empresas como, por ejemplo, Papa John's, Pizza Hut, Domino's Pizza, D'nnos Pizza, entre otras, y el gran número de pizzerías, trattorias y restaurantes, en general, que también ofrecen el producto.

La demanda de pizzas de cada empresa depende de varios factores, no solo de su calidad y precio, sino también de otras variables como el servicio, la ubicación de los locales, la comodidad de las instalaciones, entre otros factores vinculados a la calidad de bienes y servicios. Es muy probable que también esté en función de la imagen de la empresa y la responsabilidad social empresarial. La presente investigación pretende explorar acerca de las principales variables que influyen en la decisión de compra de los consumidores de pizza, en un grupo de jóvenes universitarios de carreras de negocios y, en particular, analizar si variables como la imagen y la responsabilidad social influyen en la decisión de compra. 
La selección del perfil del estudiante -universitarios de carreras de negocios- se realizó por conveniencia, dado que el estudio pretende indagar los patrones de consumo en un grupo de estudiantes que, por la naturaleza de sus estudios universitarios, deben ser sensibles y valorar aspectos como la responsabilidad social o la imagen de marca. De igual forma, se seleccionó la Universidad del Pacífico por el perfil de esta universidad, que ofrece carreras universitarias vinculadas a los negocios.

Asimismo, la selección de una marca específica de pizza se realizó sobre la base de un sondeo efectuado a diez estudiantes, con el fin de determinar la empresa de mayor recordación en el rubro.

\section{Objetivos y alcances del estudio}

Aunque el objetivo central general de la investigación es identificar las variables que influyen en la intención de compra de pizzas en estudiantes universitarios de carreras de negocios, en el presente estudio se seleccionó una marca específica de pizzas -como resultado de un sondeo a estudiantes universitarios- y las preguntas del cuestionario estuvieron dirigidas a evaluar los patrones de consumo referidos a la marca seleccionada.

En ese sentido, los resultados de la presente investigación están relacionados con los factores que influyen en la compra de una marca de pizza en particular, sin poder generalizar las conclusiones a todo el sector. Si bien este aspecto representa una limitación del presente estudio, en futuras investigaciones se puede extender el análisis a todo el sector y plantear, además, un estudio concluyente.

Vinculado al propósito del estudio, los objetivos específicos son:

- Identificar los factores y las variables que influyen en la intención de compra de una marca específica de pizzas en el segmento elegido.

- Formular un modelo de causalidad de los factores y las variables identificadas.

- Analizar en qué medida los factores y las va- riables del modelo explican la intención de compra de una marca específica de pizzas en el segmento elegido.

Asimismo, el objetivo del estudio se centra en medir los factores que influyen en la compra de pizzas solo en los locales. Es decir, no se incluye la compra bajo la modalidad de delivery.

\section{Marco teórico}

De acuerdo con los objetivos del estudio, se exploró acerca de modelos y estudios empíricos que permiten identificar los factores y las variables que influyen en la intención de compra de pizzas u otros negocios que podrían tener factores de decisión de compra similares. En particular, se identificaron cuatro tipos de factores o "constructos» que pueden influir en la intención de compra de pizzas: «calidad» (referida a la mezcla de bienes y servicios), "precio» (referido al precio en sí y otras variables asociadas), «responsabilidad social empresarial»e «imagen corporativa». A continuación, se detallan los principales sustentos y variables identificadas en cada constructo.

\section{Variables del factor "calidad»}

Respecto de este factor, aunque el término «calidad» es definido por varios autores y bajo diferentes perspectivas, en la presente investigación se consideraron dos propuestas ampliamente difundidas en la teoría administrativa: (a) el modelo conceptual de calidad de servicios y el cuestionario «SERVQUAL», propuesto por Parasuraman, Zeithmal y Berry (1988); y (b) la propuesta de «creación de valor» de Kaplan y Norton (2000), que incluye aspectos de calidad del producto $y$ del servicio, además de otros aspectos como la imagen y las relaciones de la organización. De esta forma, el constructo "calidad» está referido a la calidad de los bienes y servicios en conjunto, que permite identificar los atributos de valor de las pizzas (Kaplan y Norton, 2000) y los del servicio (Parasuraman, Zeithmal y Berry, 1985).

Según el modelo conceptual de calidad de servicios (Parasuraman, Zeithaml y Berry, 1985), la 
calidad de un servicio es el resultado de cinco gap o brechas, siendo una de ellas la diferencia entre el «servicio percibido» y las «expectativas del servicio». A su vez, en un inicio se plantearon diez factores o determinantes de la percepción de calidad: acceso al servicio, comunicación, competencia, cortesía, credibilidad, confianza, responsabilidad, seguridad, elementos tangibles del servicio y empatía.
Asociado al modelo de calidad, los autores proponen el cuestionario «SERVQUAL» (Parasuraman, Zeithaml y Berry, 1988) para medir la percepción de la calidad de un servicio mediante un conjunto de 22 ítems, estructurados en cinco dimensiones: elementos tangibles, fiabilidad, capacidad de respuesta, seguridad y empatía. En el cuadro 1, se detallan los 22 atributos de calidad.

\section{Cuadro 1}

\section{f́tems del cuestionario SERVQUAL}

\section{Elementos tangibles}

1. La empresa tiene equipos de apariencia moderna.

2. Las instalaciones físicas de la empresa de servicios son visualmente atractivas.

3. Los empleados de la empresa de servicios tienen apariencia pulcra.

4. Los elementos materiales (folletos, estado de cuenta y similares) son visualmente atractivos.

\section{Fiabilidad}

5. Cuando la empresa de servicios promete hacer algo en cierto tiempo, lo hace.

6. Cuando un cliente tiene un problema, la empresa muestra un sincero interés en solucionario.

7. La empresa realiza bien el servicio la primera vez.

8. La empresa concluye el servicio en el tiempo prometido.

9. La empresa de servicios insiste en mantener registros exentos de errores.

\section{Capacidad de respuesta}

10. Los empleados comunican a los clientes cuándo concluirá la realización del servicio.

11. Los empleados de la empresa ofrecen un servicio rápido a sus clientes.

12. Los empleados de la empresa de servicios siempre están dispuestos a ayudar a sus clientes.

13. Los empleados nunca están demasiado ocupados para responder a las preguntas de sus clientes.

\section{Seguridad}

14. El comportamiento de los empleados de la empresa de servicios transmite confianza a sus clientes.

15. Los clientes se sienten seguro en sus transacciones con la empresa de servicios.

16. Los empleados de la empresa de servicios son siempre amables con los clientes.

17. Los empleados tienen conocimientos suficientes para responder a las preguntas de los clientes.

\section{Empatía}

18. La empresa de servicios da a sus clientes una atención individualizada.

19. La empresa de servicios tiene horarios de trabajo convenientes para todos sus clientes.

20. La empresa de servicios tiene empleados que ofrecen una atención personalizada a sus clientes.

21. La empresa de servicios se preocupa por los mejores intereses de sus clientes.

22. La empresa de servicios comprende las necesidades específicas de sus clientes. 
Por otra parte, se puede identificar los atributos de calidad a partir del modelo genérico propuesto por Kaplan y Norton (2000), que trata de explicar la creación de valor para el cliente (véase el gráfico 1).

Por ejemplo, respecto de la característica «funcionalidad» de la pizza, es posible identificar la variable «variedad y tamaño de la pizza»; mientras que el «sabor y la calidad de los insumos» representa una variable relevante del rubro «calidad»; y el atributo «tiempo de espera» se despliega del rubro «tiempo». Los rubros «precio», «imagen» y «relaciones» pueden diferenciarse del concepto de calidad y contemplarse como otros factores.

Luego de analizar la aplicación de ambas alternativas al caso de las pizzas, se consolidaron las variables del factor "calidad», referido tanto a la calidad de las pizzas en sí como a la calidad del servicio, las que se muestran en el cuadro 2.

\section{Gráfico 1}

\section{La propuesta de valor añadido para el cliente}

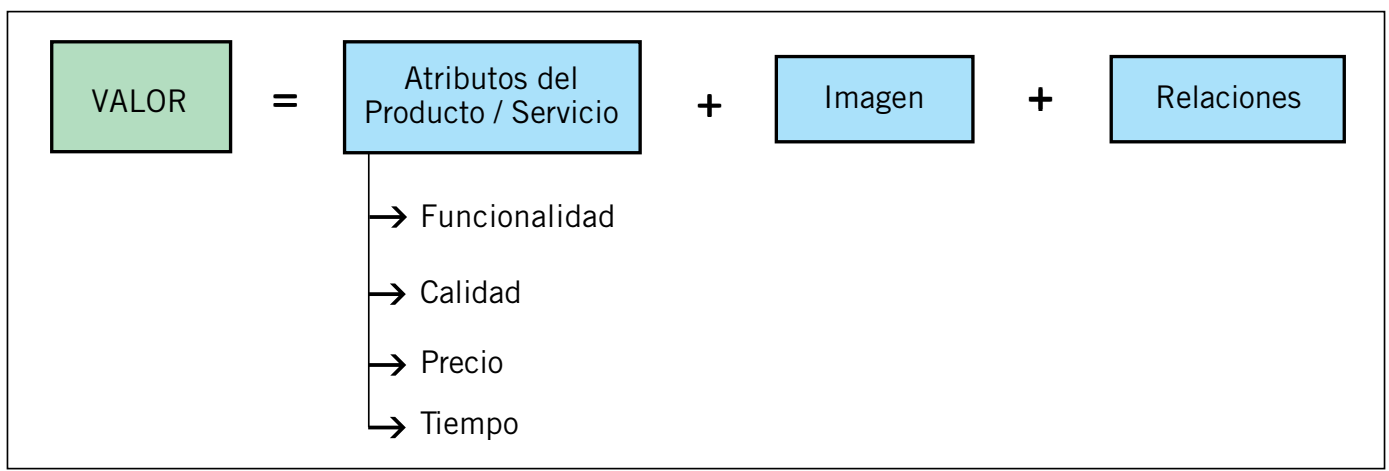

Fuente: Basado en Kaplan y Norton, 2000: Pág. 93

Elaboración propia.

\section{Cuadro 2}

Atributos del factor «calidad»

\begin{tabular}{|l|l|l|l|}
\hline \multirow{4}{*}{} & Q1 & 1 & Sabor y calidad de los insumos \\
\cline { 2 - 4 } & Q2 & 2 & Variedad y tamaño de pizzas \\
\cline { 2 - 4 } & Q3 & 3 & Trato y cortesía del personal \\
\cline { 2 - 4 } & Q4 & 4 & Presentación del personal \\
\cline { 2 - 4 } & Q5 & 5 & Conocimiento del personal \\
\cline { 2 - 4 } CALIDAD & Q7 & 6 & Atención personalizada \\
\cline { 2 - 4 } & Q8 & 7 & Tiempo de espera \\
\cline { 2 - 4 } & Q9 & 9 & Información de elementos visuales \\
\cline { 2 - 4 } & Q10 & 10 & Respuesta a reclamos y sugerencias \\
\cline { 2 - 4 } & Q11 & 11 & Ubicación de locales \\
\cline { 2 - 4 } & Q12 & 12 & Atractividad de locales \\
\cline { 2 - 4 } & Q13 & 13 & Estado de hornos y equipos \\
\cline { 2 - 4 } & Q14 & 14 & NIVEL DE SATISFACCIÓN - CALIDAD \\
\hline
\end{tabular}

Elaboración propia. 
Como se puede apreciar, se han identificado trece variables que originan catorce preguntas de la encuesta, teniendo presente que se planteará una pregunta de control para medir el nivel de satisfacción global por la calidad de la pizza.

Variables del factor «imagen»

De acuerdo con el modelo genérico de Kaplan y Norton (2000), la «imagen» es un factor relevante que agrega valor al cliente. Al revisar estudios referidos a este factor, se encontró una propuesta que plantea una escala de medición de la imagen basada en tres dimensiones: imagen funcional, imagen afectiva y reputación (Martínez, Montaner y Pina, s. f.).

\section{Cuadro 3}

\section{Dimensiones del factor «imagen»}

\section{Imagen funcional:}

- Los productos son de alta calidad.

- Los productos presentan características que otras marcas no tienen.

- Es muy poco probable que consumir esta marca ocasione problemas o imprevistos.

- Los productos de la competencia suelen ser más baratos.

Imagen afectiva:

- Es una marca que despierta simpatía.

- Esta marca transmite una personalidad que la diferencia de las marcas competidoras.

- Comprar esta marca dice algo sobre la clase de persona que eres.

- Tengo una imagen del tipo de personas que compran esta marca.

- Es una marca que no decepciona a sus clientes.

\section{Reputación:}

- Es una de las mejores marcas del sector.

- Es una marca comprometida con la sociedad.

- Es una marca muy consolidada en el mercado.
Si se consideran estas variables y se adaptan al producto pizzas, se tendrán las siguientes variables del factor «imagen»:

\section{Cuadro 4}

Atributos del factor «imagen»

\begin{tabular}{|c|c|c|c|}
\hline \multirow{6}{*}{$\begin{array}{l}\text { ్ㅀ } \\
\text { 。్ } \\
\text { ్ㅡ }\end{array}$} & Q15 & 15 & Características diferenciales \\
\hline & Q16 & 16 & Garantía \\
\hline & Q17 & 17 & Confianza de la marca \\
\hline & Q18 & 18 & Estatus de la marca \\
\hline & Q19 & 19 & Prestigio de la marca \\
\hline & Q20 & 20 & Nivel de satisfacción - imagen \\
\hline
\end{tabular}

Elaboración propia.

En este factor o "constructo», se han identificado cinco variables que originan seis preguntas de la encuesta, considerando que se planteará una pregunta de control para medir el nivel de satisfacción por la imagen de la empresa.

\section{Variables del factor "responsabilidad social»}

Según el modelo genérico de Kaplan y Norton (2000), las «relaciones» constituyen un factor que agrega valor al cliente. Este concepto no se refiere solamente a las relaciones cliente-empresa, también puede conceptualizarse desde la perspectiva de la «responsabilidad social» (RS). Es decir, en qué medida el cliente valora las relaciones de la empresa con otros agentes, como los proveedores, la sociedad en general, el medioambiente, el Gobierno o, inclusive, con otras empresas competidoras.

Luego de efectuar una revisión de estudios referidos a la «responsabilidad social», se decidió por la propuesta de Bigne-Alcañiz y Currás-Pérez (2008), quienes realizan un exhaustivo estudio y plantean los atributos para medir la influencia de la responsabilidad social corporativa en la intención de compra de productos (véase el cuadro 5). 


\section{Cuadro 5}

\section{Atributos del factor «responsabilidad social»}

Imagen de responsabilidad social:

- [Marca] Está concienciada por asuntos medioambientales.

- [Marca] Cumple con sus responsabilidades sociales.

- $\quad$ Marca] Devuelve algo de lo que ha recibido a la sociedad.

- Creo que [marca] actúa con el interés de la sociedad en mente.

- [Marca] actúa de forma socialmente responsable.

- [Marca] integra las contribuciones filantrópicas en sus actividades de negocio.

Imagen de habilidad corporativa:

- Creo que [marca] ofrece unos productos con una buena relación calidad-precio.

- [Marca] tiene una gran experiencia en productos de [ámbito/sector].

- [Marca] ofrece productos de calidad.

Identificación con la empresa:

- La imagen que tengo de [marca] encaja con la imagen que tengo de mí mismo.

- Mi forma de ser pega con lo que percibo de [marca].

- Me parezco a lo que pienso que [marca] representa.

- Soy similar a cómo percibo a [marca].

Intención de compra:

- La próxima vez que necesite un comprar algún [tipo de producto], lo haré de [marca].

- Es muy probable que en el futuro compre algún producto de [marca].

- Definitivamente, compraré algún producto de [marca].

Fuente: Bigne-Alcañiz y Currás-Pérez, 2008: 18.

Sobre la base del primer rubro de atributos de «Imagen de responsabilidad social», en el cuadro 6 se detallan los atributos considerados en este factor.
Por lo tanto, en este factor se han identificado cuatro variables observables que dan lugar a cinco preguntas de la encuesta, teniendo presente que, al igual que en los factores anteriores, se

\section{Cuadro 6}

Atributos del factor «responsabilidad social»

\begin{tabular}{|c|l|l|l|}
\hline \multirow{4}{*}{$\begin{array}{c}\text { Responsabilidad } \\
\text { social }\end{array}$} & Q21 & 21 & Responsabilidad medioambiental \\
\cline { 2 - 4 } & Q22 & 22 & Responsabilidad social \\
\cline { 2 - 4 } & Q23 & 23 & Responsabilidad con clientes \\
\cline { 2 - 4 } & Q24 & 24 & Responsabilidad con proveedores \\
\cline { 2 - 4 } & Q25 & 25 & NIVEL DE SATISFACCIÓN - RS \\
\hline
\end{tabular}


planteará una pregunta de control para medir el nivel de satisfacción por la responsabilidad social de la empresa. Es importante resaltar que en este constructo no se está midiendo la responsabilidad social de la empresa referida a la investigación, sino la reputación o la percepción que tienen los clientes respecto de la responsabilidad social de la empresa.

\section{Variables del factor «precio»}

En cuanto al constructo "precio», se analizaron las distintas ofertas de pizzas en Lima para identificar las variables vinculadas a este factor. En este contexto, se observó que, además del precio de lista, las empresas presentan promociones 0 descuentos y ventas por un conjunto o pack de productos, denominados «combos», que también representan una forma de descuento. Sobre la base de este escenario, en el cuadro 7 se detaIlan las variables consideradas en este factor.

\section{Cuadro 7}

\section{Atributos del factor "precio»}

\begin{tabular}{|c|l|l|l|}
\hline \multirow{4}{*}{$\begin{array}{c}\text { Precio y } \\
\text { promociones }\end{array}$} & Q26 & 26 & Precio de lista \\
\cline { 2 - 4 } & Q27 & 27 & Responsabilidad social \\
\cline { 2 - 4 } & Q28 & 28 & Combos atractivos \\
\cline { 2 - 4 } & Q29 & 29 & NIVEL DE SATISFACCIÓN - RS \\
\hline
\end{tabular}

Elaboración propia.

\section{Cuadro 9}

\section{Metodología de investigación propuesta}

\begin{tabular}{|c|c|c|c|}
\hline \multicolumn{2}{|c|}{ Fase } & Fuentes de información / Metodología \\
\hline Fase 1 & $\begin{array}{c}\text { Construcción del modelo } \\
\text { teórico (tentativo) }\end{array}$ & $\begin{array}{l}\text { Construcción del modelo teórico, a partir de las relaciones de causalidad entre } \\
\text { los factores y las variables identificadas en la revisión del marco teórico. }\end{array}$ \\
\hline \multirow{2}{*}{ Fase 2 } & $\begin{array}{c}\text { Diseño y aplicación } \\
\text { de encuestas }\end{array}$ & $\begin{array}{l}\text { Diseño del cuestionario, sobre la base de las variables y preguntas identificadas } \\
\text { como relevantes en el marco teórico. } \\
\text { Aplicación de encuestas a jóvenes de la Universidad del Pacífico, en Lima. En } \\
\text { este estudio, en un nivel exploratorio, se eligió el marco muestral y la muestra } \\
\text { por conveniencia, teniendo presente que los resultados representan un sondeo } \\
\text { del público encuestado. Es decir, es muy probable que no sean representativos } \\
\text { de la población total de jóvenes universitarios de Lima. }\end{array}$ \\
\hline Análisis estadístico y 2 & $\begin{array}{l}\text { Aplicación de análisis factorial y ecuaciones estructurales para identificar los } \\
\text { factores y variables estadísticamente significativas que permiten explicar la } \\
\text { intención de compra de pizzas. }\end{array}$ \\
\hline
\end{tabular}

Elaboración propia.
En este factor se han identificado tres variables observables que originan cuatro preguntas de la encuesta, considerando que se planteará una pregunta de control para medir el nivel de satisfacción global por el precio.

\section{Variables dependientes del Modelo «intención de compra»}

Por último, se identificó la «intención de compra» como la variable dependiente que se desea medir, tal como lo plantearon los autores Bigne-Alcañiz y Currás-Pérez (2008). Asimismo, se planteó una última variable de control relacionada con el nivel de consumo de pizzas que, aunque no representa una variable, puede conducir a algún análisis de correlación relevante (véase el cuadro 8).

\section{Cuadro 8}

Atributos del factor «intención de compra»

\begin{tabular}{|c|c|c|l|}
\hline \multirow{2}{*}{$\begin{array}{c}\text { Actitud de } \\
\text { compra }\end{array}$} & Q30 & 30 & Intención de compra \\
\cline { 2 - 4 } & Q31 & 31 & Nivel de consumo \\
\hline
\end{tabular}

Elaboración propia.

\section{Metodología de la investigación}

El presente estudio representa un ensayo de corte científico que, a partir del marco teórico y de una encuesta aplicada en un nivel exploratorio, analiza e identifica los factores y las variables estadísticamente significativas para explicar la intención de compra de pizzas. En este contexto, en el cuadro 9 se resume la metodología por utilizar. 
Factores que explican la intención de compra de pizzas en jóvenes universitarios de carreras de negocio

\section{Fase 1: Modelo teórico tentativo}

En función de las variables observables identificadas, así como de las relaciones entre los constructos planteados en otros estudios, se propone un modelo tentativo (véase el gráfico 2).

\section{Gráfico 2}

\section{Modelo teórico (tentativo)}

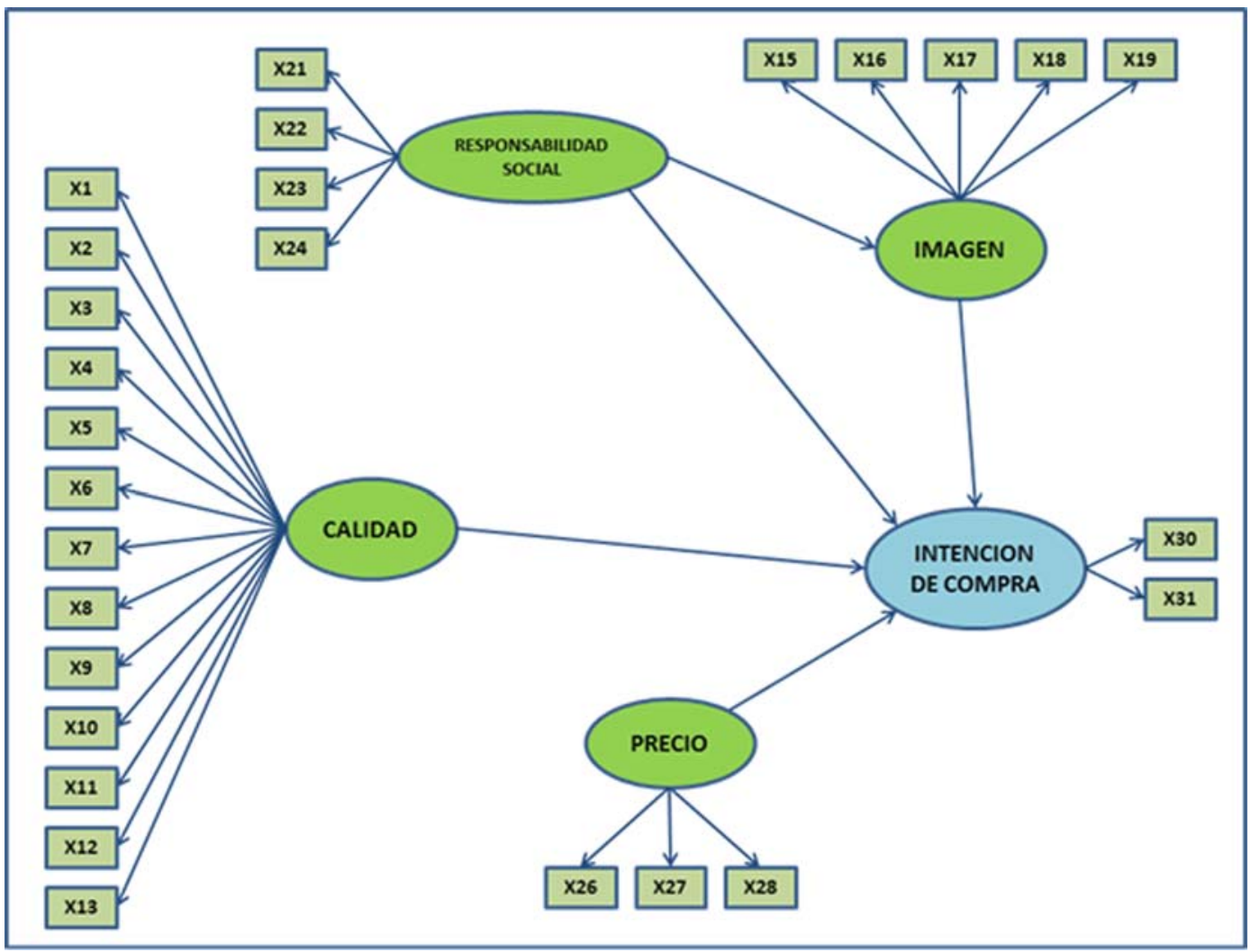

Elaboración propia.

En el gráfico se puede apreciar que las variables «Xi» representan las variables identificadas en el marco teórico, que corresponden a los factores: «calidad» (véase el cuadro 2), «imagen» (véase el cuadro 4), «responsabilidad social» (véase el cuadro 6) y "precio» (véase el cuadro 7); mientras que la variables $" \mathrm{X}_{30}$ " $\mathrm{y}$ « $\mathrm{X}_{31}$ » representan la «intención de compra» y el «nivel de consumo», respectivamente.

\section{Fase 2: Diseño y aplicación de encuesta}

De acuerdo con las variables observables identificadas, se planteó un cuestionario de 31 preguntas, clasificadas por grupos que corresponden a los constructos o factores. Asimismo, con el fin que los encuestados tengan claro lo que deben evaluar, se plantearon frases que cumplieron el papel de «reactores». A continuación, se detaIlan los reactores: 
Factores que explican la intención de compra de pizzas en jóvenes universitarios de carreras de negocio

Constructo «calidad»
Q1 Estoy muy satisfecho por el sabor y la calidad de los insumos de la pizza.
Q2 Estoy muy satisfecho con la variedad y el tamaño de las pizzas.
Q3 Los empleados tienen un trato amable, cortés y transmiten confianza a sus clientes.
Q4 El uniforme y la presentación de los empleados es adecuada.
Q5 Los empleados conocen los productos y responden adecuadamente las preguntas de los clientes.
Q6 Los empleados ofrecen una atención personalizada y responden a los requerimientos específicos de los clientes.
Q7 El tiempo de espera para ordenar y recibir la pizza es el adecuado.
Q8 Los elementos visuales son atractivos y contienen información clara del producto y del servicio.
Q9 Los empleados responden de manera adecuada ante cualquier reclamo o sugerencia de los clientes.
Q10 Los horarios de atención al público son adecuados.
Q11 Los locales de atención están bien adecuados.
Q12 Los locales son visualmente atractivos, cómodos y limpios.
Q13 Los hornos y equipos son modernos y están en buen estado.
Q14 En general, valore el nivel de calidad.

\section{Constructo «imagen»}
Q15 Las pizzas presentan características que las hacen diferentes de las demás.
Q16 La pizza es de garantía, siempre está en las mejores condiciones.
Q17 La marca es de confianza, no decepciona a sus clientes.
Q18 Comprar esta marca da un cierto estatus.
Q19 Es una de las mejores marcas del sector, está muy consolidada en el mercado.
Q20 En general, valore la imagen de la marca.

\section{Constructo «responsabilidad social»}
Q21 La empresa se preocupa de aspectos medioambientales.
Q22 La empresa cumple con sus responsabilidades sociales, actúa de manera socialmente responsable.
Q23 La empresa es socialmente responsable con sus clientes.
Q24 La empresa es socialmente responsable con sus proveedores y otros stakeholders.
Q25 En general, valore la responsabilidad social de la empresa. 


\section{Constructo "precio»}

Q26 El precio de las pizzas es adecuado.

Q27 La empresa ofrece buenas promociones y descuentos.

Q28 La empresa ofrece combos variados y atractivos.

Q29 En general, valore el precio y la oferta de la empresa.

Constructo «intención de compra» $y$ «consumo»

Q30 La próxima vez que compre una pizza, ¿pediré la marca $X$ ?

Q31 El último mes, ¿cuántas veces ha comprado la pizza?

Por otro lado, para medir las variables planteadas mediante reactivos, se presentó una escala de Liker con las siguientes cinco opciones cerradas:

Totalmente en desacuerdo / En desacuerdo / Indiferente / De acuerdo /

Totalmente de acuerdo

En el caso de las preguntas filtro de medición global de constructos, se consideró las siguientes opciones:

Muy malo / Malo / Regular / Bueno / Muy buena

En el caso de las preguntas relacionadas con el constructo «intención de compra» y "consumo», cada una tuvo sus propias opciones cerradas:

\section{Q30 La próxima vez que compre una pizza, ¿pediré la marca $X$ ?}

Poco probable / No sé / Quizás / Lo más probable / De todas maneras

\section{Q31 El último mes, ¿cuántas veces ha comprado la pizza? \\ 0 / 1 / 2 / 3 / 4 o más}

Asimismo, al inicio de la encuesta se plantearon variables de control: Carrera universitaria, Distrito de residencia y Sexo, con el fin de obtener una buena distribución de la muestra e información del perfil de los encuestados. La encuesta se aplicó a estudiantes universitarios de la Universidad del Pacífico, entre el 10 y el 15 de noviembre de 2010 , en los salones de clase (escogidos por conveniencia para encuestar a estudiantes de Administración y Economía, principalmente) que se presentan en el cuadro 10.

\section{Cuadro 10}

Muestra de la encuesta realizada a los jóvenes universitarios de la Universidad del Pacífico

\begin{tabular}{|l|c|l|}
\hline \multicolumn{1}{|c|}{ Curso } & Encuestados & \multicolumn{1}{|c|}{ Fecha de encuesta } \\
\hline Administración de Operaciones (A) & 24 & Miércoles, 10 de noviembre, 2010 \\
Administración de Operaciones (B) & 23 & Miércoles, 10 de noviembre, 2010 \\
Economía General I (C ) & 27 & Lunes, 15 de noviembre, 2010 \\
Economía General I (B) & 31 & Lunes, 15 de noviembre, 2010 \\
Matemática I (A) & 27 & Jueves, 11 de noviembre, 2010 \\
Matemática I (H) & 23 & Jueves, 11 de noviembre, 2010 \\
\cline { 2 - 3 } & 155 & \multicolumn{2}{|l}{} \\
\cline { 2 - 2 } & &
\end{tabular}


Como puede apreciarse, en total se obtuvieron 155 encuestas, siendo la composición de la muestra la siguiente:

- El $62,6 \%$ de los encuestados pertenece a la carrera de Administración; un 21,3\%, a la carrera de Economía y el resto, a otras carreras como Contabilidad, Ingeniería Empresarial o Derecho Empresarial.
- El 36,1\% de los encuestados pertenece al género masculino y el $63,9 \%$, al femenino.

- El $72,3 \%$ de encuestados reside en la zona 7 de Lima; el 14,2\%, en la zona 6; mientras que el $5,8 \%$ proviene de la zona 8 y otro $5,8 \%$ reside en otras zonas. Estas zonas geográficas de residencia muestran la composición de los encuestados según el nivel socioeconómico, para lo cual se tuvo en cuenta la clasificación de distritos realizada para Lima que se presenta en el cuadro 10.

\section{Cuadro 11}

\section{Zonas geográficas de Lima y niveles socioeconómicos}

\section{Agrupación de distritos en 10 zonas geográficas en base a criterios de proximidad geográfica, características socioculturales, económicas y de estilo de vida}

\begin{tabular}{|c|c|c|c|c|}
\hline Zona 1 & Zona 3 & Zona 6 & Zona 7 & Zona 9 \\
\hline $\begin{array}{l}\text { Ventanilla } \\
\text { Puente Piedra } \\
\text { Comas } \\
\text { Carabayllo }\end{array}$ & $\begin{array}{l}\text { San Juan de } \\
\text { Lurigancho }\end{array}$ & $\begin{array}{l}\text { Jesús María } \\
\text { Lince } \\
\text { Pueblo Libre } \\
\text { Magdalena } \\
\text { San Miguel }\end{array}$ & $\begin{array}{l}\text { Miraflores } \\
\text { San Isidro } \\
\text { San Borja } \\
\text { Surco } \\
\text { La Molina }\end{array}$ & $\begin{array}{l}\text { Villa El Salvador } \\
\text { Villa María del Triunfo } \\
\text { Lurín } \\
\text { Pachacamac }\end{array}$ \\
\hline Zona 2 & Zona 4 & Zona 5 & Zona 8 & Zona 10 \\
\hline $\begin{array}{l}\text { Independencia } \\
\text { Los Olivos } \\
\text { San Martín de Porras }\end{array}$ & $\begin{array}{l}\text { Cercado } \\
\text { Rímac } \\
\text { Breña } \\
\text { LaVictoria }\end{array}$ & $\begin{array}{l}\text { Ate } \\
\text { Chaclacayo } \\
\text { Lurigancho } \\
\text { Santa Anita } \\
\text { San Luis } \\
\text { El Agustino }\end{array}$ & $\begin{array}{l}\text { Surquillo } \\
\text { Barranco } \\
\text { Chorrillos } \\
\text { San Juan de Miraflores }\end{array}$ & $\begin{array}{l}\text { Callao } \\
\text { Bellavista } \\
\text { La Perla } \\
\text { La Punta } \\
\text { Carmen de la Legua }\end{array}$ \\
\hline
\end{tabular}

\begin{tabular}{|c|c|c|c|c|}
\hline \multicolumn{5}{|c|}{ NIVEL SOCIOECONÓMICO (NSE) Y PRINCIPALES ZONAS DE RESIDENCIA } \\
\hline \multicolumn{5}{|c|}{ Nivel socioeconómico } \\
\hline $\begin{array}{c}\text { NSE A } \\
\text { ALTO/MEDIO } \\
\text { ALTO }\end{array}$ & $\begin{array}{l}\text { NSE B } \\
\text { MEDIO }\end{array}$ & $\begin{array}{c}\text { NSE C } \\
\text { BAJO } \\
\text { SUPERIOR }\end{array}$ & $\begin{array}{l}\text { NSE D } \\
\text { BAJO } \\
\text { INFERIOR }\end{array}$ & $\begin{array}{c}\text { NSE E } \\
\text { MARGINAL }\end{array}$ \\
\hline $\begin{array}{c}\text { Zonas } \\
6 \text { y } 7\end{array}$ & $\begin{array}{c}\text { Zonas } \\
6 \text { y } 7\end{array}$ & $\begin{array}{l}\text { Zonas } \\
2,4,8 \text { y } 10\end{array}$ & $\begin{array}{l}\text { Zonas } \\
3,5,8 \text { y } 9\end{array}$ & $\begin{array}{l}\text { Zonas } \\
1,2,5 \text { y } 9\end{array}$ \\
\hline $\begin{array}{l}\text { La Molina } \\
\text { Miraflores } \\
\text { Magdalena } \\
\text { Barranco } \\
\text { San Isidro } \\
\text { San Miguel } \\
\text { San Borja }\end{array}$ & $\begin{array}{l}\text { Surco } \\
\text { Los Olivos } \\
\text { La Molina } \\
\text { Cercado } \\
\text { Miraflores } \\
\text { Magdalena } \\
\text { Barranco } \\
\text { Jesús María } \\
\text { San Isidro } \\
\text { San Miguel } \\
\text { Lince } \\
\text { San Borja } \\
\text { Pueblo Libre }\end{array}$ & $\begin{array}{l}\text { San Martín de Porres } \\
\text { Comas } \\
\text { Los Olivos } \\
\text { San Juan de Miraflores } \\
\text { Callao } \\
\text { Chorrillos } \\
\text { La Victoria } \\
\text { Carmen Legua } \\
\text { Santa Anita } \\
\text { Carabayllo } \\
\text { La Perla } \\
\text { Barranco } \\
\text { Breña } \\
\text { Bellavista } \\
\text { San Luis }\end{array}$ & $\begin{array}{l}\text { Ate } \\
\text { San Juan Lurigancho } \\
\text { San Martín de Porres } \\
\text { Villa El Salvador } \\
\text { San Juan de Mir.aflores } \\
\text { Chorrillos } \\
\text { La Victoria } \\
\text { El Agustino } \\
\text { Rímac } \\
\text { Puente Piedra } \\
\text { Lurigancho } \\
\text { Villa María del Triunfo } \\
\text { Independencia } \\
\text { Bellavista } \\
\text { Lurín }\end{array}$ & $\begin{array}{l}\text { Ate } \\
\text { San Juan de Lurigancho } \\
\text { Villa El Salvador } \\
\text { Villa María del Triunfo } \\
\text { Puente Piedra } \\
\text { Lurigancho } \\
\text { Ventanilla } \\
\text { Pachacamac } \\
\text { Carabayllo } \\
\text { Independencia }\end{array}$ \\
\hline
\end{tabular}


Factores que explican la intención de compra de pizzas en jóvenes universitarios de carreras de negocio

Fase 3: Análisis estadístico y validación del modelo

El análisis estadístico se realizó por etapas, con el fin de validar los resultados en el interior de cada factor o constructo, y después integrar los elementos en un único modelo.

- Validación de variables de cada constructo: análisis factorial

En el modelo tentativo, cada constructo o factor está conformado por varias variables. Antes de iniciar el análisis factorial se calculó el coeficiente Alfa de Cronbach (á), que debe ser mayor a 0,7 (á > 0,7) para aprobar el test de confiabilidad de los datos.

En el análisis factorial aplicado a cada factor o constructo se planteó de manera consecutiva este análisis, hasta que las variables expliquen o se puedan resumir en solo un "factor», que se valida cuando se logran los siguientes tres objetivos:
- Índice KMO > 0,9 (nivel excelente)

- Prueba de Esfericidad con nivel de significancia á < 0,05

- Porcentaje de varianza explicada del único factor $>50 \%$

Si en una prueba en particular no se logra validar el análisis factorial, se vuelve a realizar este análisis pero eliminando la variable de menor significancia, que en esta técnica se denomina "comunalidad» (es decir, se elimina la variable de menor comunalidad).

A continuación se resumen los principales indicadores obtenidos en el análisis factorial aplicado, de manera consecutiva, a cada uno de factores o constructos.

Factor «calidad»

Test de confiabilidad de datos

Alfa de Cronbach: á $=0,876 \quad$ (á $>0,7$ )

\begin{tabular}{|c|c|c|c|c|c|c|}
\hline \multirow{2}{*}{ Variable } & \multicolumn{6}{|c|}{ Análisis factorial con un solo componente } \\
\hline & Prueba 1 & Prueba 2 & Prueba 3 & Prueba 4 & Prueba 5 & Prueba 6 \\
\hline Número de variables & 13 & 12 & 11 & 1110 & 109 & 8 \\
\hline $\mathrm{x}$ & $\mathrm{X}$ & $\mathrm{x}$ & $\mathrm{x}$ & $\mathrm{x}$ & $\mathrm{X}$ & $\mathrm{x}$ \\
\hline $\mathrm{x} 2$ & $x$ & $x$ & & & & \\
\hline $\mathrm{x} 3$ & $x$ & $\mathrm{x}$ & $x$ & $x$ & $\mathrm{X}$ & $x$ \\
\hline $\mathrm{X} 4$ & $x$ & $x$ & $x$ & $x$ & $x$ & $x$ \\
\hline$x 5$ & $x$ & $x$ & $x$ & $x$ & $x$ & $x$ \\
\hline$x 6$ & $x$ & $x$ & $x$ & $x$ & $x$ & $x$ \\
\hline $\mathrm{X7}$ & $x$ & & & & & \\
\hline $\mathrm{x} 8$ & $x$ & $x$ & $x$ & $x$ & $x$ & \\
\hline X9 & $x$ & $x$ & $x$ & $x$ & $x$ & $x$ \\
\hline $\mathrm{X} 10$ & $x$ & $x$ & $x$ & & & \\
\hline $\mathrm{X} 11$ & $x$ & $x$ & $x$ & $x$ & & \\
\hline $\mathrm{X} 12$ & $x$ & $x$ & $x$ & $x$ & $x$ & $x$ \\
\hline $\mathrm{X} 13$ & $x$ & $x$ & $x$ & $x$ & $x$ & $x$ \\
\hline KMO(Validar: KMO > 0,9) & 0,890 & 0,899 & 0,905 & 0,905 & 0,900 & 0,892 \\
\hline $\begin{array}{c}\text { Test de Esfericidad(Validar: } \\
\text { á }<0,05)\end{array}$ & 0,000 & 0,000 & 0,000 & 0,000 & 0,000 & 0,000 \\
\hline $\begin{array}{l}\% \text { Varianza explicada } \\
\text { (Validar: VE > 50\%) }\end{array}$ & $41,38 \%$ & $43,69 \%$ & $45,66 \%$ & $47,24 \%$ & $49,13 \%$ & $51,72 \%$ \\
\hline Mínima comunalidad & 0,163 & 0,260 & 0,350 & 0,355 & 0,341 & \\
\hline Variable por eliminar & $x 7$ & $x 2$ & $\mathrm{X} 10$ & $\mathrm{X} 11$ & $x 8$ & \\
\hline
\end{tabular}

Conclusión: eliminar las variables X2, X7, X8, X10 y X11. Aunque no se logran los límites «ideales»para validar el modelo $(K M O=0,892)$, los indicadores resultan muy aproximados a los valores permisibles. 
Factores que explican la intención de compra de pizzas en jóvenes universitarios de carreras de negocio

Factor «imagen»

Test de confiabilidad de datos

Alfa de Cronbach: á $=0,787 \quad$ (á $>0,7)$

\begin{tabular}{|c|c|c|}
\hline \multirow{2}{*}{ Variable } & \multicolumn{2}{|c|}{ Análisis factorial con un solo componente } \\
\hline & Prueba 1 & Prueba 2 \\
\hline Número de variables & 5 & 4 \\
\hline $\mathrm{x} 15$ & $x$ & $x$ \\
\hline $\mathrm{X} 16$ & $x$ & $x$ \\
\hline $\mathrm{x} 17$ & $x$ & $x$ \\
\hline $\mathrm{x} 18$ & $x$ & \\
\hline $\mathrm{x} 19$ & $x$ & $x$ \\
\hline $\begin{array}{c}\text { KMO } \\
\text { (Validar: } \mathrm{KMO}>0,9)\end{array}$ & 0,804 & 0,772 \\
\hline $\begin{array}{l}\text { Test de Esfericidad } \\
\text { (Validar: á < 0,05) }\end{array}$ & 0.000 & 0.000 \\
\hline $\begin{array}{l}\text { \% Varianza explicada } \\
\text { (Validar: VE > 50\%) }\end{array}$ & $55,86 \%$ & $61,51 \%$ \\
\hline Mínima comunalidad & 0,439 & \\
\hline Variable por eliminar & $\mathrm{x} 18$ & \\
\hline
\end{tabular}

Conclusión: no eliminar ninguna variable, dado que el índice «KMO» baja mucho al eliminar la variable X18. Aunque no se logran los límites «ideales» para validar el modelo (KMO =0,804), los indicadores resultan muy aproximados a los valores permisibles.

Factor «responsabilidad social»

Test de confiabilidad de datos

Alfa de Cronbach: á $=0,860 \quad$ (á $>0,7)$

\begin{tabular}{|c|c|c|}
\hline \multirow{2}{*}{ Variable } & \multicolumn{2}{|c|}{ Análisis factorial con un solo componente } \\
\cline { 2 - 3 } & Prueba 1 & Prueba 2 \\
\hline Número de variables & 5 & 4 \\
\hline X21 & X & X \\
X23 & X & X \\
X24 & X & \\
\hline KMO & 0,738 & 0,658 \\
\hline (Validar: KMO > 0,9) & 0.000 & 0.000 \\
\hline Test de Esfericidad & & \multirow{2}{*}{$78,208 \%$} \\
\hline Validar: á < 0,05) & $70,598 \%$ & \\
\hline \% Varianza & 0,587 & \\
\hline explicada (Validar: VE $>50 \%)$ & X24 & \\
\hline Mínima comunalidad & & \\
\hline Variable por eliminar & & \\
\hline
\end{tabular}


Factores que explican la intención de compra de pizzas en jóvenes universitarios de carreras de negocio

Conclusión: no eliminar ninguna variable, en vista de que el índice «KMO» baja mucho al eliminar la variable X24. Si bien no se logran los límites «ideales» para validar el modelo ( $K M O=0,738$ ), los indicadores resultan muy aproximados a los valores permisibles.

Factor «precio»

Test de confiabilidad de datos

Alfa de Cronbach: á $=0,804 \quad$ (á $>0,7$ )

\begin{tabular}{|c|c|c|}
\hline \multirow{2}{*}{ Variable } & \multicolumn{2}{|c|}{ Análisis factorial con un solo componente } \\
\cline { 2 - 3 } & Prueba 1 & Prueba 2 \\
\hline Número de variables & 3 & 2 \\
\hline X26 & X & X \\
X28 & X & X \\
\hline KMO & 0,691 & 0,500 \\
\hline (Validar: KMO > 0,9) & 0.000 & 0.000 \\
\hline Test de Esfericidad & & $83,911 \%$ \\
\hline (Validar: á < 0,05) & $72,266 \%$ & \\
\hline Varianza explicada & 0,641 & \\
\hline Malidar: VE > 50\%) & X26 & \\
\hline Variable por eliminar & & \\
\hline
\end{tabular}

Conclusión: no eliminar ninguna variable, pues el índice «KMO» baja mucho al eliminar la variable X26. Pese a que no se logran los límites «ideales» para validar el modelo (KMO =0,738), los indicadores resultan relativamente cerca a los valores permisibles.

- Validación de relaciones variablesconstructos: análisis de variables de control

Como en la encuesta se incluyeron preguntas de control relacionadas con la «satisfacción global» para cada uno de los constructos, de manera complementaria al análisis factorial es posible medir el coeficiente de correlación entre la satisfacción global de cada constructo (medido a través de una sola pregunta) y la valoración del constructo obtenido, de manera directa, del análisis factorial (variable latente o ficticia creada por el modelo).

En el cuadro 11 se resume este análisis.

\section{Cuadro 11}

Coeficiente de correlación entre la satisfacción global de cada constructo y la valoración del constructo

\begin{tabular}{|c|c|c|}
\hline Constructo (análisis factorial) & Variable de control & Coeficiente de correlación \\
\hline Calidad & P14(Calidad) & 0,54 \\
\hline Imagen & P20(Imagen) & 0,66 \\
\hline Responsabilidad social & P25(RS) & 0,69 \\
\hline Precio & P29(Precio) & 0,79 \\
\hline
\end{tabular}


Tal como se puede apreciar en el cuadro, tres coeficientes de correlación tienen valores relativamente altos y aceptables: 0,66, 0,69 y 0,79, correspondientes a los constructos imagen, responsabilidad social y precio, respectivamente. Si bien el valor del coeficiente de correlación del constructo calidad $(0,54)$ no es tan alto como los anteriores, también podría considerarse «aceptable» porque este constructo tiene un número alto de variables observables, que de alguna manera aumenta la probabilidad de errores de valoración por parte de los encuestados.
Por su parte, el nivel de correlación entre la intención de compra y el consumo resultó muy bajo, motivo por el cual se eliminó esta última variable (pregunta 31 de la encuesta).

Luego de haber realizado el análisis factorial, y haber analizado las variables de control, el modelo teórico tentativo quedó depurado tal como se muestra en el gráfico 3.

\section{Gráfico 3}

\section{Depuración del modelo teórico tentativo basado en el análisis factorial}

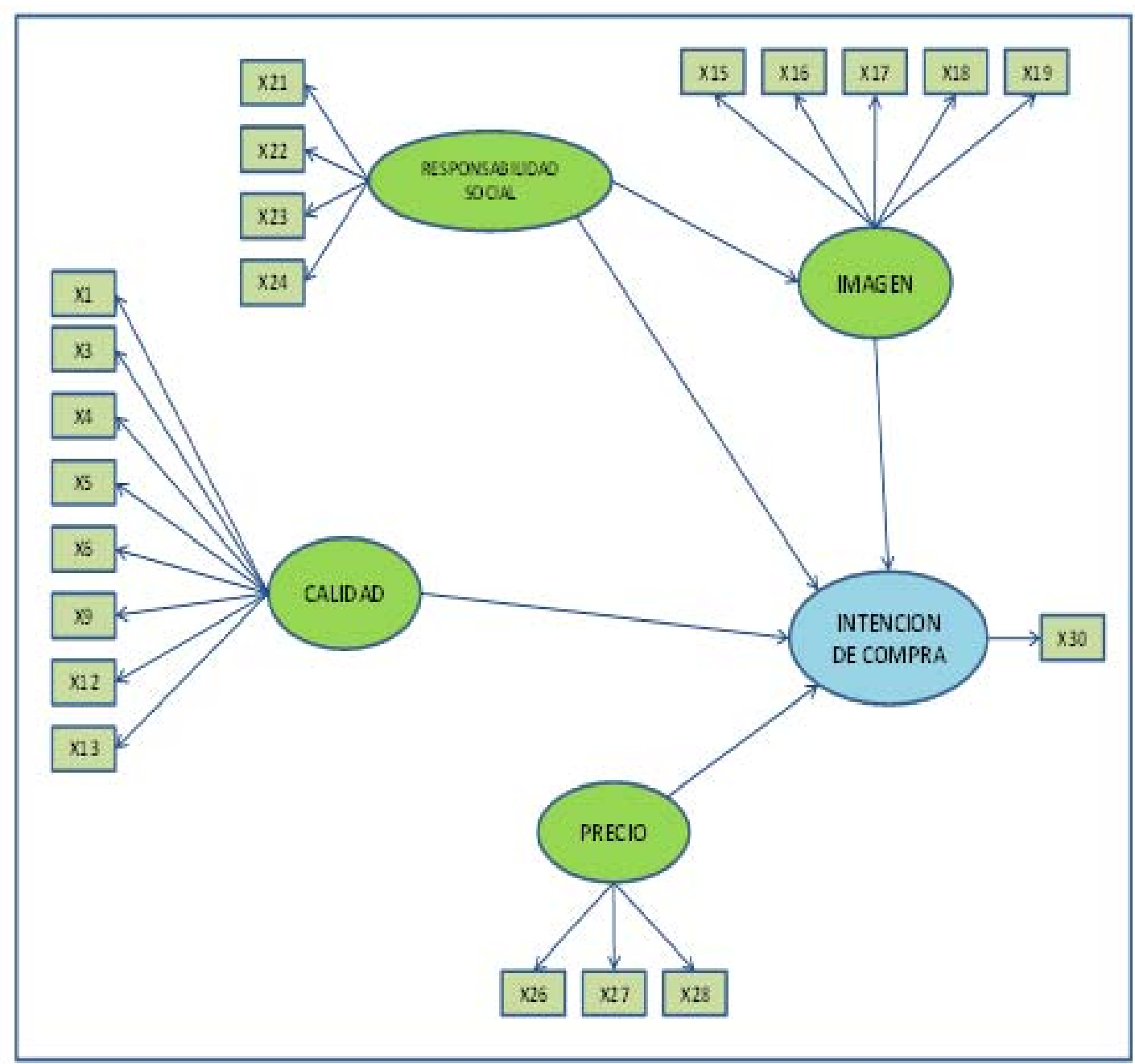


- Análisis de correlación entre constructos y regresión múltiple

Con el fin de analizar en una primera instancia la relación entre constructos, se midió el coeficiente de correlación entre los constructos relacionados teóricamente. En el gráfico 4 se muestran los resultados obtenidos (los valores corresponden a los coeficientes de correlación).

Nótese que el constructo responsabilidad social no tiene una correlación significativa con los constructos imagen e intención de compra. Por tanto, si nos limitamos a plantear un análisis de correlación simple entre constructos, la responsabilidad social debería eliminarse del modelo. Si se efectúa la eliminación, tendríamos un modelo de regresión múltiple:

Intención de compra = f(Calidad, Imagen, Precio)

En el cuadro 12 se presenta el reporte de dicho modelo de regresión múltiple.

\section{Gráfico 4}

Análisis de correlación entre los constructos del modelo teórico tentativo

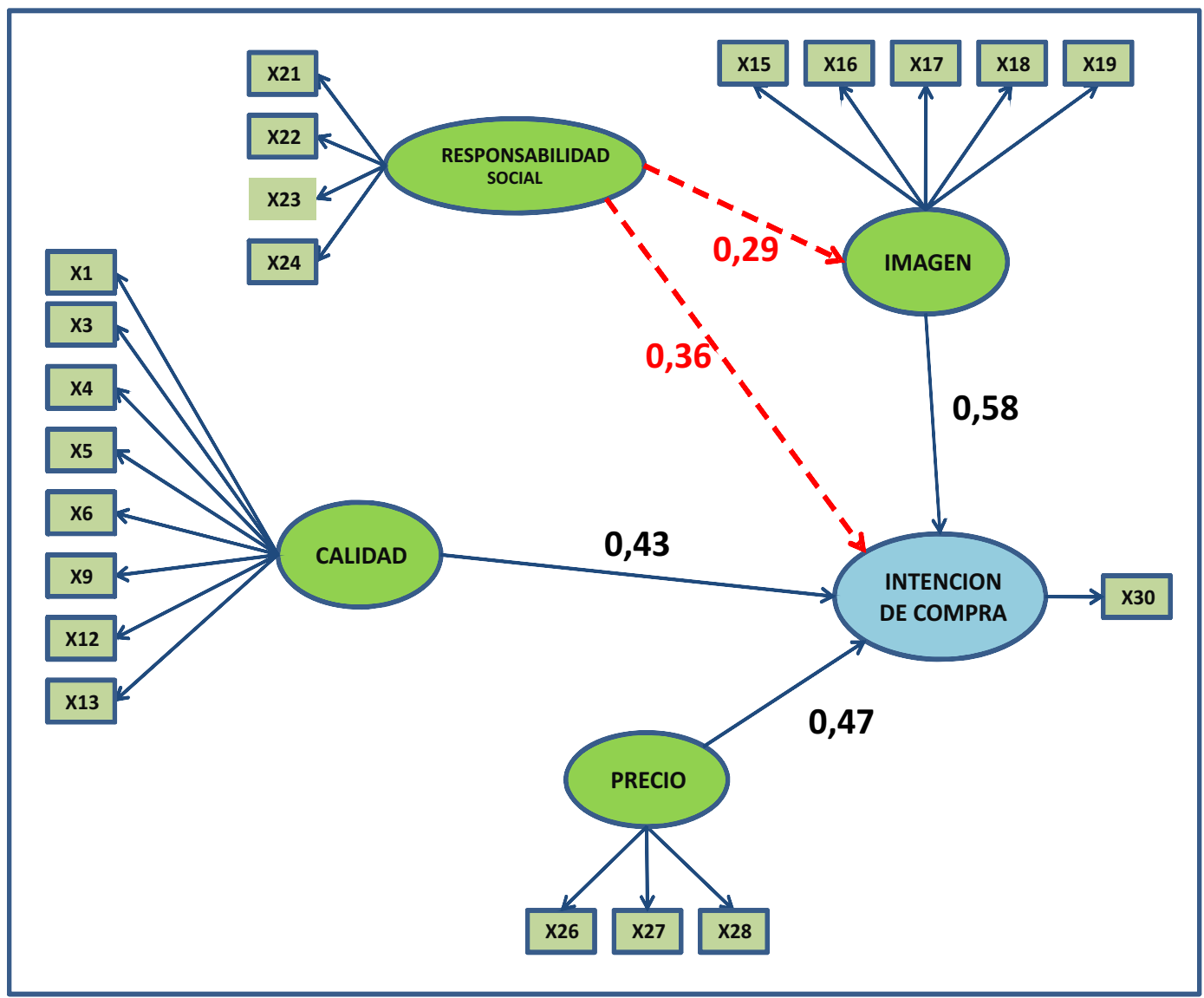

Elaboración propia. 
Cuadro 12

Reporte del modelo de regresión múltiple

\begin{tabular}{|c|c|c|c|c|c|c|c|c|}
\hline \multicolumn{4}{|c|}{ Estadísticas de la regresión } & & & & & \\
\hline \multicolumn{3}{|c|}{ Coeficiente de correlación múltiple } & 0.6579 & & & & & \\
\hline \multicolumn{3}{|c|}{ Coeficiente de determinación $\mathrm{R}^{\wedge} 2$} & 0.4328 & & & & & \\
\hline \multicolumn{3}{|l|}{$R^{\wedge} 2$ ajustado } & 0.4215 & & & & & \\
\hline \multicolumn{3}{|l|}{ Error típico } & 0.9052 & & & & & \\
\hline \multicolumn{3}{|l|}{ Observaciones } & 155 & & & & & \\
\hline \multicolumn{9}{|c|}{ ANÁLISIS DE VARIANZA } \\
\hline & & $\begin{array}{c}\text { Grados de } \\
\text { libertad }\end{array}$ & $\begin{array}{c}\text { Suma de } \\
\text { cuadrados }\end{array}$ & $\begin{array}{l}\text { Promedio de } \\
\text { los cuadrados }\end{array}$ & $\boldsymbol{F}$ & \multicolumn{2}{|c|}{$\begin{array}{c}\text { Valor } \\
\text { crítico de }\end{array}$} & \\
\hline Regresión & & 3 & 94.4142 & 31.4714 & \multirow[t]{3}{*}{38.4043} & \multicolumn{2}{|c|}{0.0000} & \\
\hline Residuos & & 151 & 123.7407 & 0.8195 & & & & \\
\hline \multirow[t]{2}{*}{ Total } & & 154 & 218.1548 & & & & & \\
\hline & Coeficientes & Error típico & Estadístico $t$ & Probabilidad & $\begin{array}{c}\text { Inferior } \\
95 \%\end{array}$ & $\begin{array}{c}\text { Superior } \\
95 \%\end{array}$ & $\begin{array}{c}\text { Inferior } \\
95.0 \%\end{array}$ & $\begin{array}{c}\text { Superior } \\
95.0 \%\end{array}$ \\
\hline Intercepción & -2.4758 & 0.5562 & -4.4511 & 0.0000 & -3.5748 & -1.3768 & -3.5748 & -1.3768 \\
\hline Variable X 1 & 0.1979 & 0.1146 & 1.7267 & 0.0863 & -0.0286 & 0.4244 & -0.0286 & 0.4244 \\
\hline Variable $\times 2$ & 0.5471 & 0.1099 & 4.9769 & 0.0000 & 0.3299 & 0.7642 & 0.3299 & 0.7642 \\
\hline Variable X 3 & 0.3840 & 0.0806 & 4.7640 & 0.0000 & 0.2247 & 0.5432 & 0.2247 & 0.5432 \\
\hline
\end{tabular}

Tal como se puede observar, no se logra un buen ajuste del modelo ( $R^{2}$ ajustado $=0,42$ ), aunque los otros parámetros son adecuados y aceptables (el valor de $\mathrm{F}$ y el valor crítico de F, la probabilidad de cada variable y los signos de la ecuación de regresión múltiple). En la ecuación de regresión múltiple se puede apreciar que X2 = Imagen es el factor que más incide en la «intención de compra» (coeficiente de la ecuación $=0,55$ ), seguido de la variable $\mathrm{X} 3=$ Precio (coeficiente de la ecuación $=0,38$ ) y, por último, el factor $\mathrm{X} 1=$ Calidad (coeficiente de la ecuación $=0,20$ ). Este ranking de importancia en la «intención de compra» coincide con el orden de mayor a menor de los coeficientes de correlación (véase estos coeficientes en el gráfico 4).
- Análisis de correlación entre constructos y regresión múltiple

Con el fin de perfeccionar el modelo, se planteó un modelo de ecuaciones estructurales, desarroIlado mediante el software Lisrel, especializado para estos casos. En un primer intento se trabajó con el modelo teórico tentativo, pero no logró validarlo por sus bajos índices de ajuste. Este resultado era de esperarse, en vista del análisis previo efectuado por medio de los coeficientes de correlación.

Sin embargo, a partir de los reportes y sugerencias de los reportes del Lisrel, se planteó un modelo alternativo que sí fue validado (véanse los gráfico 5 y 6 ). 
Factores que explican la intención de compra de pizzas en jóvenes universitarios de carreras de negocio

\section{Gráfico 5}

Modelo alternativo planteado en el software Lisrel

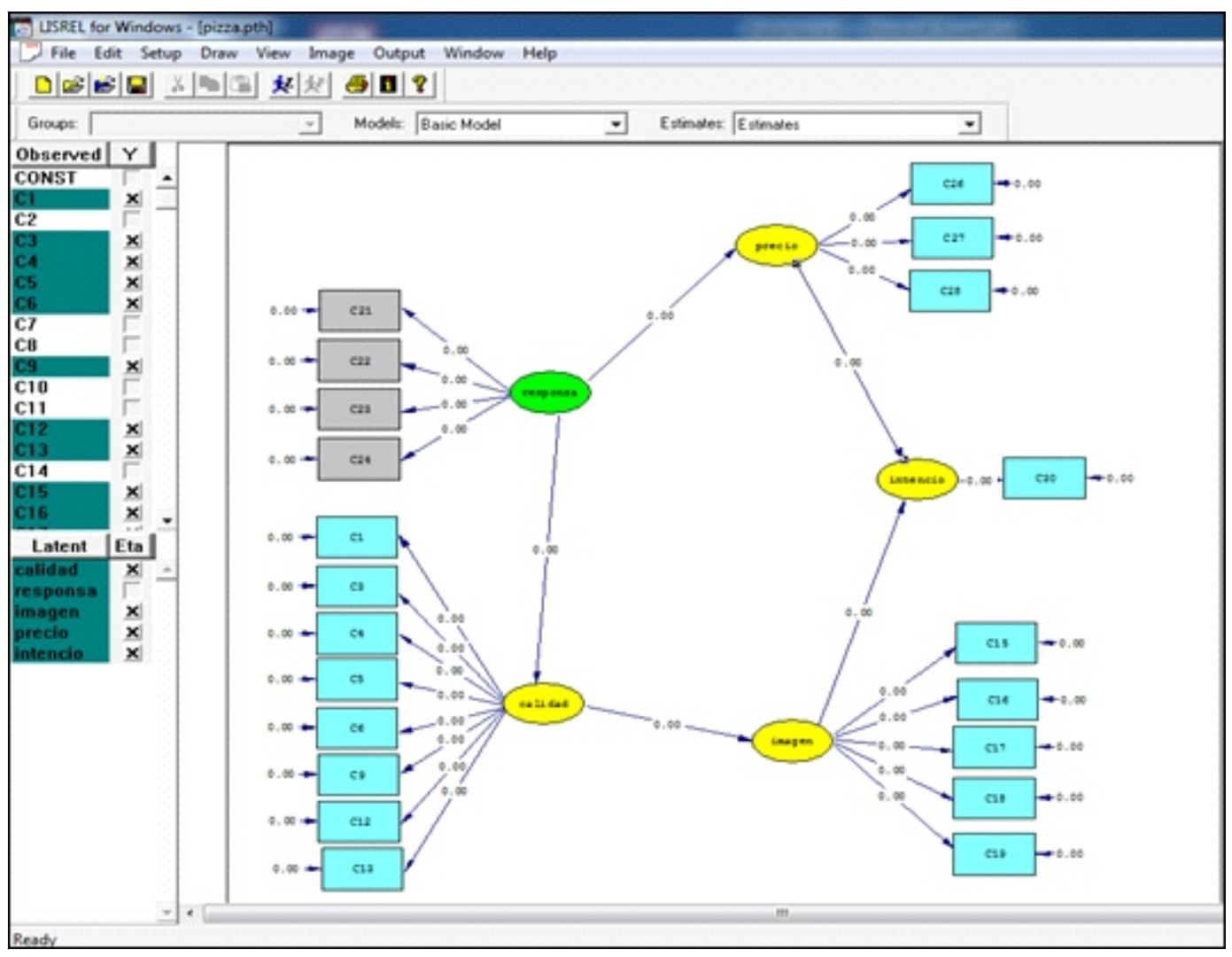

Elaboración propia. 


\section{Gráfico 6}

Solución del modelo alternativo en el software Lisrel

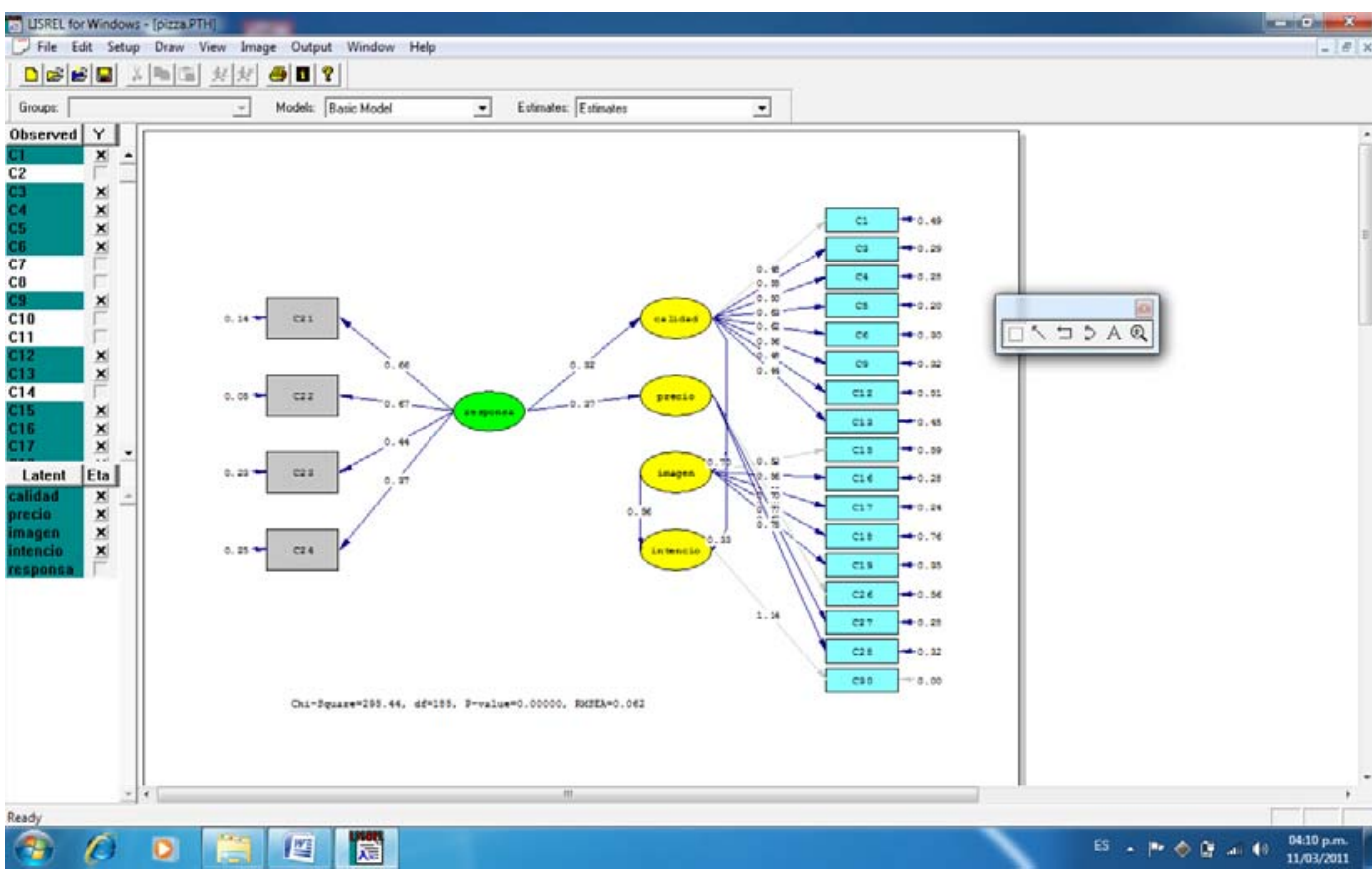

Elaboración propia.

Los principales índices de ajuste del modelo son:

Root Mean Square Error of Approximation (RMSEA) $=0,062$

Normed Fit Index (NFI) $=0,91$

Non-Nomed Fit Index (NNFI) $=0,96$

Parsimony Normed Fit Index $(\mathrm{PNFI})=0,81$

Comparative Fit Index $(\mathrm{CFI})=0,97$

A modo de resumen, en el gráfico 7 se detalla el modelo definitivo. 
Gráfico 7

Modelo definitivo

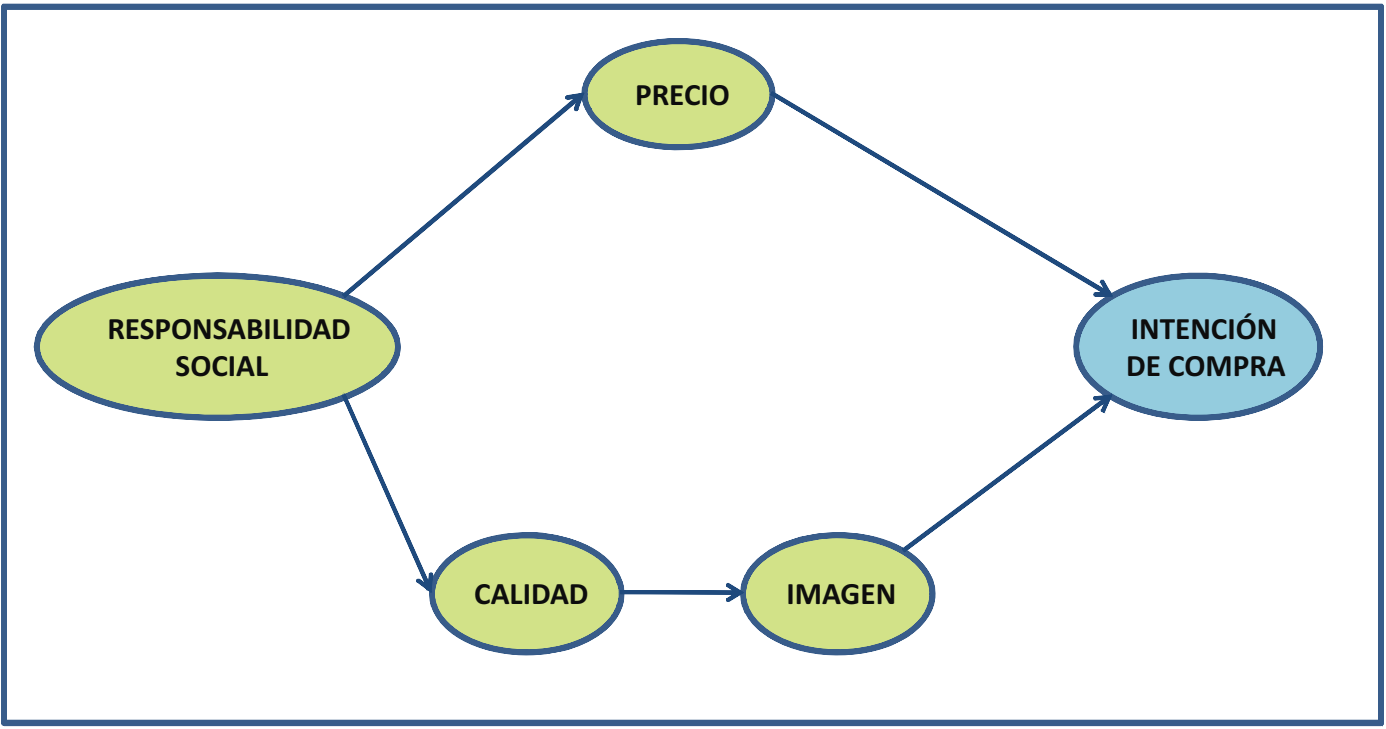

Elaboración propia.

\section{Resultados y conclusiones}

Según los hallazgos encontrados, a continuación se resumen los resultados y las conclusiones más relevantes de la investigación:

- En general, los datos obtenidos de la encuesta son confiables, pues todos los coeficientes Alfa de Cronbanch obtenidos fueron mayores a 0,7 en cada uno de los constructos: calidad (á = 0,876 ); imagen (á $=0,787$ ); responsabilidad social (á $=0,860$ ) y precio (á $=0,804)$. En este criterio, las respuestas de las preguntas asociadas a la «calidad» son las más confiables, seguidas de los datos obtenidos de «responsabilidad social», «precio» y, por último, «imagen».

- Los siguientes atributos, y en ese orden, explican el factor o constructo "calidad» (se ha establecido el orden de importancia según el coeficiente de correlación entre cada variable y el nivel de satisfacción global):

- Sabor y calidad de insumos

- Respuesta a reclamos y sugerencias
- Atractividad de locales

- Conocimiento del personal

- Atención personalizada

- Trato y cortesía del personal

- Estado de hornos y equipos

- Presentación del personal

Por el contrario, los siguientes factores no influyen de manera significativa en la percepción de calidad:

- Variedad y tamaño de pizzas

- Tiempo de espera

- Información de elementos visuales

- Horarios de atención

- Ubicación de locales

- Los siguientes atributos, y en ese orden, explican el factor o constructo «imagen»:

- Prestigio de la marca

- Confianza de la marca 
- Garantía

- Características diferenciales

- Estatus de la marca

- Los siguientes atributos, y en ese orden, explican el factor o constructo «responsabilidad social»:

- Responsabilidad social

- Responsabilidad medioambiental

- Responsabilidad con proveedores

- Responsabilidad con clientes

- Los siguientes atributos, y en ese orden, explican el factor o constructo «precio»:

- Precio

- Promociones y descuentos

- Combos atractivos

- La «responsabilidad social» influye, de manera significativa, en el "precio» y en la "calidad». A su vez, la «calidad» influye en la «imagen»; mientras que el "precio» y la «imagen» influyen en la «intención de compra»:

- De manera directa o indirecta, todas los constructos involucrados en el estudio (responsabilidad social, precio, calidad e imagen) influyen en la «intención de compra». En particular, la «responsabilidad social» influye de manera directa en la percepción de «calidad» y de "precio», y estos elementos influyen a su vez en la intención de compra.

- Los siguientes factores, y en ese orden, explican la «intención de compra de pizzas»:

- Imagen

- Precio

- Calidad

- Responsabilidad social

- Los resultados representan un sondeo realizado, en un nivel exploratorio, a un grupo de jóvenes universitarios de la Universidad del Pacífico, quienes estudian carreras de negocios. Por lo tanto, estos resultados no pueden generalizarse a toda la población de jóvenes universitarios de Lima ni a otros segmentos del mercado.

- De igual forma, los hallazgos encontrados en la intención de compra de una marca específica de pizzas no pueden generalizarse a otras marcas ni a otros productos y sectores.

- Los resultados del presente estudio, aunque solo representan un sondeo, llevan a reflexiones para continuar con futuras investigaciones en esta línea. 


\section{Referencias}

ASOCIACIÓN PERUANA DE EMPRESAS DE INVESTIGACIÓN DE MERCADOS (APEIM)

2007 «Niveles socioeconómicos 2007-2008». En: APEIM. <http://www.apeim.com.pe/images/ APEIMNSE2007_2008.pdf>.

BIGNE-ALCAÑIZ, Enrique y CURRÁS-PÉREZ, Rafael 2008 « ¿Influye la imagen de responsabilidad social enla intención de compra? El papel de la identificación del consumidor de la empresa». En: Universia Business Review, Nº 19, pp. 10-23. <http://ubr.universia.net/pdfs_web/ UBR0032008010.pdf>.

GONZÁLEZ, Hugo

2009 «Escala SERVQUAL». En: Calidad \& Gestión. Fecha de consulta: 15/10/2010. <http://calidad-gestion.com.ar/rec_gratuitos/articulos/ escala_servqual.pdf $>$.

KAPLAN, Robert S. y David P. NORTON

2000 El cuadro de mando integral (The Balanced Scorecard). $2^{\mathrm{a}}$ ed. Barcelona: Ediciones Gestión 2000.
MARTÍNEZ S., Eva; Teresa MONTANER G. y José M. PINA P.

(s. f.). «Una escala de medición de la imagen de marca aplicada a la estrategia de extensión de marca». En: ESC Europe Fecha de consulta: 20/10/ 2010. <http://www.escp-eap.net/conferences/ marketing/2005_cp/Materiali/Paper/Fr/ MARTINEZ_\%2OMONTANER_PINA..pdf>

PARASURAMAN, A.; Valarie ZEITHAML, V. y Leonard BERRY

1988 «SERVQUAL: A Multiple Item Scale for Measuring Consumer Perceptions of Service Quality». En: Journal of Retailing, vol. 64 , N 1, pp. 12-40.

1985 «A Conceptual Model of Service Quality and Its Implications for Future Research». En: Journal of Marketing, vol. 49, N 4, pp. 4150. 